\title{
Nanoscale chemical imaging of reversible photoisomerization of an azobenzene-thiol self-assembled monolayer by tip-enhanced Raman spectroscopy
}

\author{
Journal Article \\ Author(s): \\ Zheng, Liqing (iD; Wang, Xing; Shao, Feng; Hegner, Martin; Zenobi, Renato \\ Publication date: \\ 2018-01-22
}

Permanent link:

https://doi.org/10.3929/ethz-b-000220746

Rights / license:

In Copyright - Non-Commercial Use Permitted

Originally published in:

Angewandte Chemie. International Edition 57(4), https://doi.org/10.1002/anie.201710443 


\title{
Nanoscale chemical imaging of reversible photoisomerization of an azobenzene-thiol self-assembled monolayer by tip-enhanced Raman spectroscopy
}

\author{
Li-Qing Zheng, ${ }^{[1]}$ Xing Wang, ${ }^{[1]}$ Feng Shao, ${ }^{[1]}$ Martin Hegner, ${ }^{[2]}$ and Renato Zenobi ${ }^{*[1]}$
}

\begin{abstract}
An understanding of the photoisomerization mechanism at the molecular scale of molecules bound to a metal surface is required for designing photo switches at surfaces. It has remained a challenge to correlate the surface structure and isomerization of photo switches at ambient conditions. Here, the photoisomerization of a self-assembled monolayer of azobenzene-thiol molecules on an Au surface was investigated using scanning tunneling microscopy and tip-enhanced Raman spectroscopy. The unique signature of the cis isomer at $1525 \mathrm{~cm}^{-1}$ observed in tip-enhanced Raman spectra was clearly distinct from the trans isomer. Furthermore, tip-enhanced Raman images of azobenzene-thiols after ultraviolet and blue light irradiation are shown with nanoscale spatial resolution, demonstrating a reversible conformational change. Interestingly, the cis isomers of azobenzene-thiol molecules were preferentially observed at Au grain edges, which is confirmed by density functional theory calculation.
\end{abstract}

Molecular switches, which undergo molecular-scale motions upon external stimuli, are very promising for molecular electronics and high-density data storage. However, a deeper understanding of their mechanical and electronic properties at the molecular level is required for developing applications in the areas of information processing, energy and biology. ${ }^{[1-4]}$ Of the various molecular switches studied, azobenzene and its derivatives adsorbed on surfaces, one of the most thoroughly investigated classes of molecular switches, ${ }^{[5-7]}$ have attracted considerable attention in the last few years thanks to their practical applications as nanomotors ${ }^{[8]}$, adaptive imaging ${ }^{[9]}$ and energy storage devices ${ }^{[10]}$ when binding to adaptive surfaces. The photoisomerization of azobenzene can be triggered by illumination with light of the appropriate wavelength. Azobenzene undergoes trans-to-cis isomerization when irradiated with ultraviolet (UV) light (365 nm). The conformational change can be reversed by blue light $(450 \mathrm{~nm})$, but it can also be caused thermally in the dark owing to the greater thermodynamic stability of the trans isomer. ${ }^{[11-14]}$ Moreover, the isomerization can be induced mechanically ${ }^{[15]}$, thermally ${ }^{[16]}$ and

[1] Prof. Dr. Renato Zenobi, Li-Qing Zheng, Xing Wang and Feng Shao, Department of Chemistry and Applied Biosciences, Swiss Federal Institute of Technology, ETH Zurich, 8093 Zurich (Switzerland) E-mail: renato.zenobi@org.chem.ethz.ch

[2] Prof. Dr. Martin Hegner, Center for Research on Adaptive Nanostructures and Nanodevices, School of Physics, Trinity College Dublin, Dublin 2, Ireland

Supporting information for this article is given via a link at the end of the document. electrostatically ${ }^{[17]}$

Photoisomerization of different molecular switches, for example 3,3',5,5'-tetra-tert-butyl-azobenzene (TBA) ${ }^{[18]}$, 3,5-ditert-butyl-N-(3,5-di-tertbutylbenzylidene) aniline (TBI) ${ }^{[19]}$ or azobenzene thiol (ABT) ${ }^{[20]}$, adsorbed on an Au (111) surface has already been demonstrated. The mechanism governing the isomerization behavior of azobenzenes in solution, which is based on $n-\pi^{*}{ }^{[21,22]}$ or $\pi-\pi^{*}{ }^{[23]}$ electronic transition, is well understood from ultrafast time-resolved studies such as coherent resonant Raman scattering ${ }^{[24-26]}$. However, the mechanism of photoisomerization of azobenzenes is quite different when they are in direct contact with a metal surface, in contrast to the photoswitching in solution. The observation that azobenzene fails to isomerize on $\mathrm{Au}(100)$ or $\mathrm{Cu}$ (111) shows a strong substrate dependence. ${ }^{[27]}$ To date, questions about the switching mechanism of surface-bound molecules under ambient conditions remain.

For a complete understanding of the isomerization processes of $A B T$ on an $A u$ surface, it is important to provide information on both the surface structure and the molecular fingerprint with nanometer spatial resolution, so that the relationship between structure and chemical behavior can be established. ${ }^{[28-30]}$ Tip-enhanced Raman spectroscopy (TERS) is a near-field optical technique that combines the advantages of scanning probe microscopy (SPM) and plasmon-enhanced Raman spectroscopy. [31-35] In this methodology, an electrochemically etched $\mathrm{Ag}$ or $\mathrm{Au}$ tip amplifying the electromagnetic field of incident laser light enhances the Raman signal of sample molecules located close to the tip. TERS possesses high spatial resolution and high sensitivity, down to the single molecule level. ${ }^{[36,37]}$ TERS is ideally suitable to study defects and step edges, which may play a significant role in surface reactions. ${ }^{[38]}$ Only very few studies have so far addressed the photoswitching properties of metal-surface-bound $\mathrm{ABT}$ at ambient conditions, where molecular switches are used in practical applications. ${ }^{[2,39]}$ In these two works, the photoisomerization of $A B T$ and an $A B T$ derivative was probed by surface-enhanced Raman spectroscopy ${ }^{[13]}$ and scanning tunneling microscopy (STM) ${ }^{[39]}$ at ambient conditions, respectively. The reversibility ${ }^{[13]}$ and a cooperative mechanism ${ }^{[39]}$ of the photoisomerization were shown.

Here, we report the investigation of the isomerization mechanism of an ABT self-assembled monolayer (SAM) on a template stripped (TS) Au surface ${ }^{[40]}$ (Figure S1) using ambient STM-TERS, taking advantange of the strong scattering properties of ABT. The reversible photoisomerization behavior of an ABT SAM on an Au surface was demonstrated, based on the presence and absence of the characteristic vibrational mode at $1525 \mathrm{~cm}^{-1}$ of cis isomer after UV irradiation (ex situ). Furthermore, TERS maps were used to follow the isomerization 
of $A B T$, and to obtain the local information of the reactivity. Interestingly, the cis isomers of ABT molecules were preferentially observed at the grain edges of $\mathrm{Au}$. This is ascribed to the lower reaction energy of trans-to-cis isomerization at $\mathrm{Au}$ steps due to the shift of $d$ bands and different molecular orientation. These results underline that grain edges play a significant role in the photoswitching behavior of surface-bound molecules.
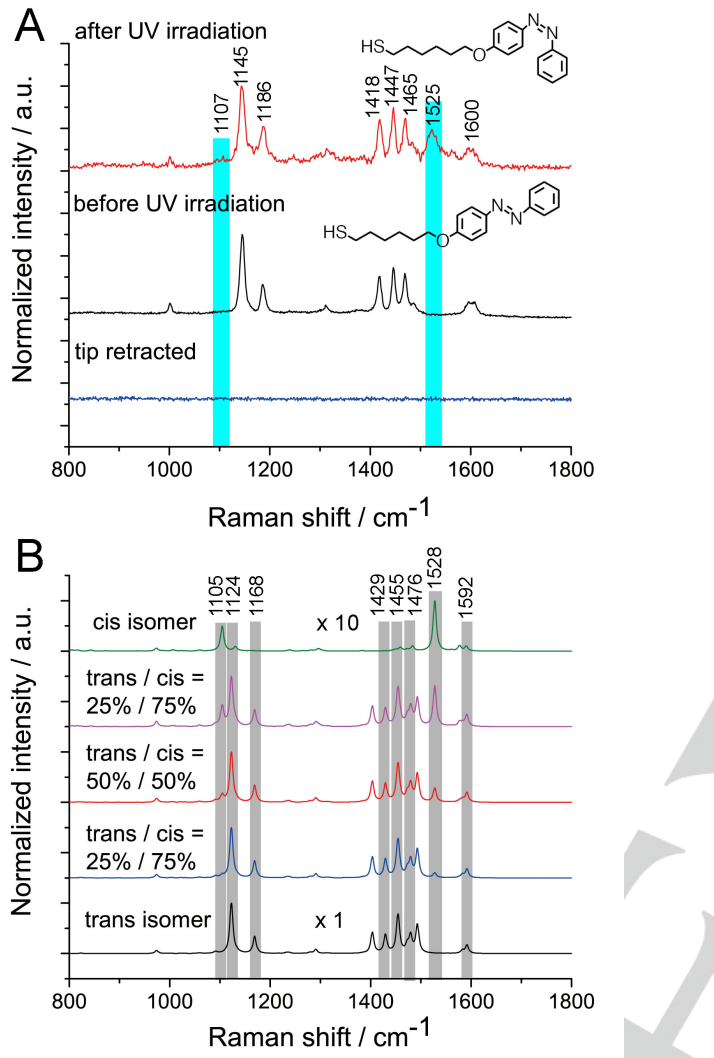

Figure 1. A. TER spectra of an ABT SAM before and after ex-situ UV irradiation for 5 mins, as well as a spectrum with the tip retracted. The blue bars indicate the Raman peak at $1525 \mathrm{~cm}^{-1}$ and $1107 \mathrm{~cm}^{-1}$ which are characteristic for the cis isomer. B. Simulated Raman spectra of the trans and cis isomers of $A B T$ with different mole fractions of the two isomers.

TER spectra of ABT SAM before and after UV irradiation for 5 mins are shown in Figure $1 A$. One can see new peaks appearing at $1525 \mathrm{~cm}^{-1}$ and $1107 \mathrm{~cm}^{-1}$ (weak band) after the sample was irradiated with $365 \mathrm{~nm}$ light. The peaks at $1525 \mathrm{~cm}^{-1}$ and $1107 \mathrm{~cm}^{-1}$ correspond to the $v_{\text {sym }}(\mathrm{N}=\mathrm{N})$ mode, and the $\mathrm{C}-\mathrm{N}$ stretching/ring breathing modes of the cis isomer, respectively. Their presence clearly indicates that trans-to-cis isomerization of $A B T$ occurred. To gain further insight into the Raman peaks observed in the TER spectra and their correlation with the transcis photoisomerization of azobenzene thiol molecules, B3LYP/6$31 \mathrm{G}^{*}$ calculations ${ }^{[13]}$ of the Raman spectra of the trans and cis isomers of $A B T$ were performed. The dependence of the spectrum on the mole fractions of the two isomers is shown in Figure 1B. The calculated frequencies were multiplied by a scaling factor of 0.97 to correct for the anharmonicity. ${ }^{[41]}$ The cis spectrum is an order of magnitude weaker than the trans spectrum. The intensity drop of the cis spectrum may be due to the loss of conjugation of the cis isomer and the consequent reduced polarizability and Raman intensity. ${ }^{[13]}$ To compensate for this intensity difference, the intensity of the cis isomer's spectrum was magnified 10 times. To have a better view of the modes, all the Raman peaks were normalized to the most intense Raman peak in each spectrum. We assigned the Raman peaks of $A B T$ according to the calculated spectra (see Figure S2). The peaks at $1145 \mathrm{~cm}^{-1}$ and $1186 \mathrm{~cm}^{-1}$ are skeletal modes with $\mathrm{C}-\mathrm{N}$ stretching and ring breathing components. The band at $1418 \mathrm{~cm}^{-1}$ corresponds to the in-plane ring bending mode with a minor contribution from the $\mathrm{N}=\mathrm{N}$ stretching mode of the trans isomer. The Raman peak at $1447 \mathrm{~cm}^{-1}$ is the $\mathrm{H}-\mathrm{C}-\mathrm{H}$ bending mode of the alkyl group. The peak at $1465 \mathrm{~cm}^{-1}$ is predominately an $\mathrm{N}=\mathrm{N}$ stretching mode of the trans isomer with minor contributions from the in-plane ring bending mode of the benzene group and the $\mathrm{H}-\mathrm{C}-\mathrm{H}$ bending mode of the alkyl group. The band at $1600 \mathrm{~cm}^{-1}$ is a $\mathrm{C}=\mathrm{C}$ stretching mode. ${ }^{[42,13]}$ Upon trans-to-cis isomerization of $\mathrm{ABT}$, the $v_{\text {sym }}(\mathrm{N}=\mathrm{N})$ frequency shifts from $1465 \mathrm{~cm}^{-1}$ to $1525 \mathrm{~cm}^{-1}$, while, the C-N stretching/ring breathing mode shifts from $1145 \mathrm{~cm}^{-1}$ to $1107 \mathrm{~cm}^{-1}$. Since the most intense Raman peak of the cis isomer is at $1525 \mathrm{~cm}^{-1}$, it can be used as a reporter peak. After UV irradiation, all of the peak intensity ratios between the trans peaks and the $1600 \mathrm{~cm}^{-1}$ band belonging to both the trans and cis isomers of the $A B T$ SAM decrease (Table S1), which also indicates the trans-to-cis isomerization of $A B T$. Based on the simulated spectra for different molar ratios of the two isomers, the TER spectrum of the ABT SAM after UV irradiation shown in Figure $1 \mathrm{~A}$ is in good agreement with a trans/cis molar ratio equal to 1:1 (see Figure S3). The low intensity of the peak at $1107 \mathrm{~cm}^{-1}$ could be due to TERS surface selection rules. ${ }^{[37]}$

The photoisomerization behavior of an ABT SAM on a semitransparent $\mathrm{Au}$ surface was first verified using UV-Vis spectroscopy. The absorbance difference at $340 \mathrm{~nm}$ of the ABT SAM before and after UV irradiation is shown in Table. S2. The absorbance at $340 \mathrm{~nm}$ decreases upon irradiation by UV light, confirming that trans-to-cis photoisomerization on the Au surface takes place. Moreover, when the irradiation time is prolonged from 5 mins to $15 \mathrm{mins}$, the value remains the same. This indicates that the steady-state trans-to-cis isomerization yield of the ABT SAM is already reached after a UV irradiation time of 5 mins. Upon irradiation with blue light for $15 \mathrm{mins}$, the absorbance at $340 \mathrm{~nm}$ recovers, indicating cis-to-trans isomerization.

The lifetime of the cis isomers on an Au surface is of great importance. The absorbance change at $340 \mathrm{~nm}$ of an ABT SAM on an Au surface after UV irradiation as a function of time is shown in Figure S4. The plot was fitted to exponential function. The thermal back reaction on the surface is dominated by firstorder kinetics with a reaction rate constant of $5.67 \times 10^{-5} \mathrm{~s}^{-1}$. The lifetime of the cis isomer on the Au surface is thus around $5 \mathrm{~h}$, which is sufficient for acquisition of a TERS map. Isomerization of $A B T$ in ethanol solution is shown in Figure S5.

TERS maps of an ABT SAM on a TS Au surface before and after irradiation of UV light were collected. Since the characteristic peak of the cis isomer is at $1525 \mathrm{~cm}^{-1}$, which is in the region where spurious peaks from carbonaceous 
decomposition products usually appear, a very low laser power and a short exposure time were used to collect the TERS maps. Figure 2 shows the TERS peak intensity ratio $\left(1525 \mathrm{~cm}^{-1}\right.$ peak / $1143 \mathrm{~cm}^{-1}$ peak) maps of the ABT SAM on the Au surface before and after UV irradiation for different times. A dark blue color corresponding to 0 in the scale bar indicates that there is no Raman peak at $1525 \mathrm{~cm}^{-1}$ in that particular region. In Figure 2A, we can barely see the cis peak. After the ABT SAM was irradiated with UV light for 5 mins, the Raman peak at $1525 \mathrm{~cm}^{-1}$ appeared much more frequently than before, indicating that part of the ABT molecules underwent a trans-to-cis isomerization. (Figure 2B) This peak is irregularly distributed, and present in $29 \%$ of all the pixels. To assess the variability of the peak at $1525 \mathrm{~cm}^{-1}$ associated with the isomerization of $A B T$, principal component analysis (PCA) on Figure 2B was performed. (Section S3) PC1 corresponds to the TER spectrum of trans isomer, while PC2 may correspond to that of trans and cis isomers. Only a very small contribution from PC3, which stems from carbon contamination, is present in the spectra. This suggests that sample decomposition is minor.
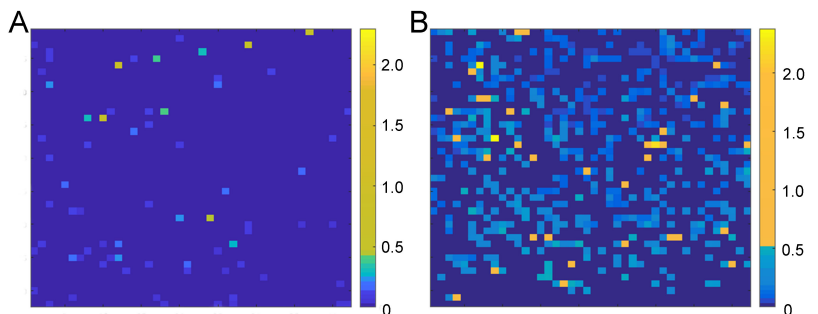

C
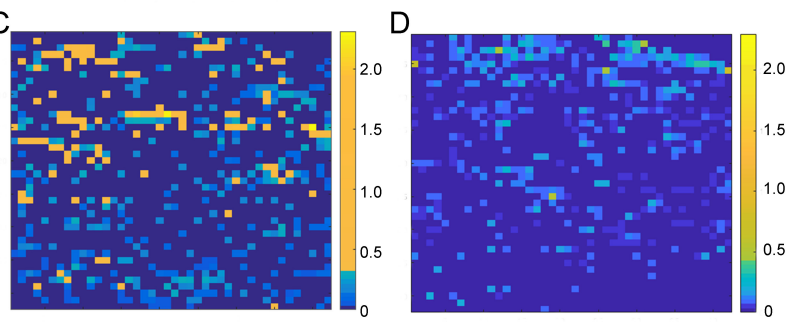

Figure 2. TERS peak intensity ratio $\left(1525 \mathrm{~cm}^{-1}\right.$ peak $/ 1143 \mathrm{~cm}^{-1}$ peak) maps of ABT SAM before (A) and after UV irradiation for 5 mins (B) and 15 mins $(C)$. The sample was prepared in the following way: $A B T$ molecules were first self-assembled on a TS Au substrate, followed by irradiation with UV light for 5 mins and 15 mins. TERS peak intensity ratio map of the ABT SAM after UV irradiation (D). The sample was prepared in the following way: ABT in ethanol was first irradiated with UV light for 5 mins and then deposited onto a TS Au substrate. The size of the maps is $200 \times 200 \mathrm{~nm}^{2}$ with a $4.8 \mathrm{~nm}$ pixel size. The acquisition time for each spectrum is $1 \mathrm{~s}$.

When the exposure time was increased to 15 mins, the presence of this peak in the whole map was slightly decreased to $26 \%$, which is a bit surprising. We interpret this to be due to the thermal back reaction during the TERS mapping (Figure $2 \mathrm{C}$ ). From the data in Figure $2 \mathrm{C}$, the spatial resolution was estimated to be around $10 \mathrm{~nm}$ based on a fullwidth at half-maximum (FWHM) analysis (Figure S8).

Another sample preparation method was tested to check the different isomerization rate of $A B T$ in ethanol solution and in the form of a SAM on the Au surface. ABT in ethanol solution was first irradiated with UV light for 5 mins, and then deposited on an Au surface, which takes ca. 120 mins. Finally, the TERS map of the ABT SAM sample was collected. (Figure 2D) The proportion of the cis peak in the whole map is $23 \%$, which is smaller than that in Figure $2 \mathrm{~B}$. This indicates that part of the cis isomers of $A B T$ molecules must have reacted back to the trans isomers, while assembly on the Au surface was carried out. When ABT molecules are bound to a metal surface, the lifetime of the cis isomers decreases, as demonstrated above.

The ABT SAM on the Au surface was irradiated with UV light for 5 mins and then irradiated with blue light for 5 mins. The TERS map and the corresponding STM image are shown in Figure 3. The fraction of pixels showing the cis peak in the whole map is $14 \%$, much smaller than that in Figure $2 \mathrm{~B}$ and Figure $2 \mathrm{C}$. This indicates that most of the cis-ABT molecules had isomerized back to the trans form. However, we clearly observe a ribbon of cis ABT located in the middle of the map, making up $72 \%$ of all pixels containing the cis peak. Interestingly, when comparing the topography collected simultaneously with the TERS map (Figure 3B), we found that this cis ribbon is situated at a place where there is an $A u$ grain edge. Moreover, the TERS peak intensity ratio $\left(1418 \mathrm{~cm}^{-1} / 1600 \mathrm{~cm}^{-1}\right)$ map is complementary to the TERS peak intensity ratio $\left(1525 \mathrm{~cm}^{-1} / 1600 \mathrm{~cm}^{-1}\right)$ map. (Figure S9) Two further peak ratio maps of ABT adsorbed at terraces and grain edges of Au before UV illumination are shown in Figure $\mathrm{S} 10$ and S11. One can barely see any peak from the cis isomer. Moreover, there is no obvious decrease of the (1418 $\mathrm{cm}^{-1} / 1600 \mathrm{~cm}^{-1}$ ) peak intensity ratio in the region where there is an Au grain edge (Figure $\mathrm{S} 10 \mathrm{~B}$ and S10C, Figure S11B and S11C), which indicates that the complementary peak intensity ratio maps shown in Figure $S 9$ is not induced by different orientation of molecules adsorbed on grain edges. Therefore, the results shown in Figure S9 clearly suggest that the cis-to-trans isomerization at the grain edge of $\mathrm{Au}$ is slower than that on the terrace.

A
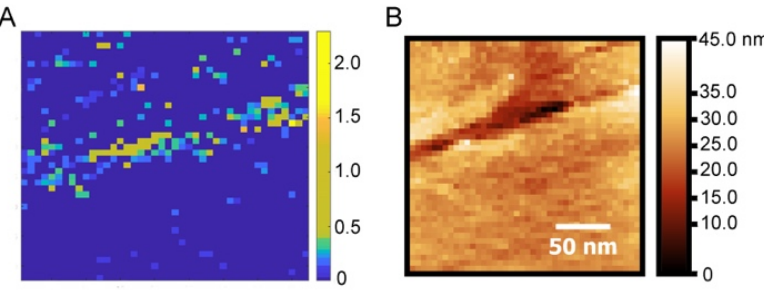

Figure 3. A. TERS peak intensity ratio $\left(1525 \mathrm{~cm}^{-1}\right.$ peak $/ 1143 \mathrm{~cm}^{-1}$ peak) map of an ABT SAM after UV irradiation for 5 mins followed by blue light irradiation for 5 mins; $B$. STM image of ABT SAM on the Au surface acquired together with the TERS map. The size of the map is $200 \times 200$ $\mathrm{nm}^{2}$ with a $4.8 \mathrm{~nm}$ pixel size. The acquisition time for each spectrum is $1 \mathrm{~s}$.

To confirm that the isomerization behavior at grain edges of $\mathrm{Au}$ is different from that on terraces, several high-resolution TERS measurements going across a terrace and a grain edge on $\mathrm{Au}$ were performed, and the results are shown in Figure 4, Figure S12 and Figure S13. We observed that the peak at $1525 \mathrm{~cm}^{-1}$ has a strong preference to be observed at Au grain edges (Figure 4B, Figure S12B and Figure S13B). Moreover, all of the peak intensity ratios between trans peaks and the $1600 \mathrm{~cm}^{-1}$ peak in the TER spectra of ABT adsorbed at the $A u$ grain edges after UV irradiation (shown in Figure $4 \mathrm{~B})$ are smaller than that of $\mathrm{ABT}$ adsorbed on Au terraces 
(Figure S14), which also indicates that trans-to-cis isomerization of $\mathrm{ABT}$ took place predominantly at the grain edge of Au.

To understand the excitation mechanism in the photoisomerization, the electronic structure of ABT SAM on the Au surface and the density of states of the Au substrate should be taken into account. Tegeder and coworkers proposed a substrate-mediated charge transfer process for the photoisomerization of TBA on an Au surface ${ }^{[7]}$. UV light first generates hot holes in the Au d-band, which relax to the top of the d-band via an Auger-decay subsequently. The hot holes undergo a charge transfer process to the HOMO of TBA, leading to the formation of transient positive ions, which may subsequently result in the isomerization of TBA. According to density functional theory (DFT) calculations (Figure S15), the energy levels of the step edge Au atoms shift to higher values compared to those of Au atoms on the terrace due to unsaturation and a decrease in inter-atom coupling ${ }^{[43]}$. The higher the d-band center, the higher the reactivity. ${ }^{[44]}$ Moreover, we calculated the total energy of trans-ABT isomers and cis-ABT isomers adsorbed on an $A u$ terrace and a 2-atom step edge, respectively. (see Figure S16) The reaction enthalpy of trans-to-cis isomerization at the 2-atom step edge is $0.61 \mathrm{eV}$, which is $13 \%$ lower than that at the terrace $(0.72 \mathrm{eV})$. Therefore, the cis isomers of $\mathrm{ABT}$ on step edges are thermodynamically more stable compared to those on terrace sites. It is fairly well established, for example, that the cis isomers of TBI are preferentially found on the herringbone reconstruction of $\mathrm{Au}$, as observed from STM images due to the slight corrugation at these sites. ${ }^{[20]}$

A

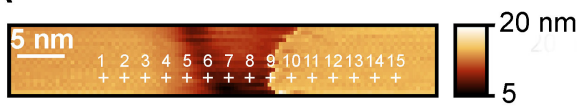

B

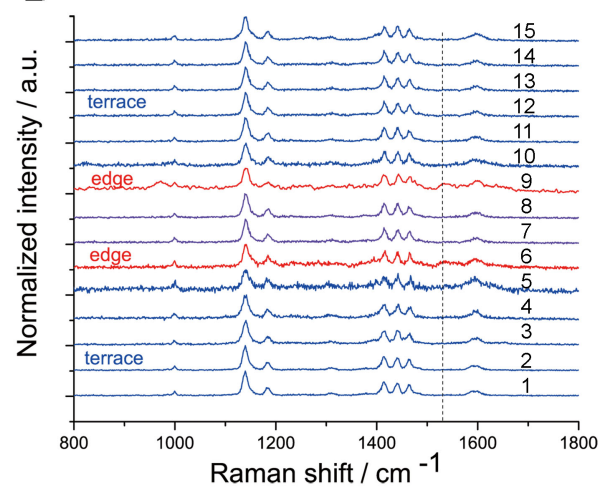

Figure 4. A. STM image of the Au surface covered with an ABT SAM after UV irradiation for 5 mins, obtained using an Ag tip; B. line-trace TER spectra along the crosses indicated in A. One spectrum was acquired every $2 \mathrm{~nm}$ of surface distance. The exposure time for per spectrum is $1 \mathrm{~s}$.

Also, because of the completely different geometry at grain edges, the molecular orientation of ABT molecules adsorbed at these sites is different from that of $A B T$ molecules adsorbed at the terraces of $\mathrm{Au}$, which could favor the trans-to-cis photoisomerization of ABT (Figure S17).

In summary, a low-cost TS Au substrate with a number of steps was fabricated and used for the investigation of photoisomerization of an ABT SAM using STM-TERS. TER spectra of ABT SAM before and after UV light illumination were collected. Based on the presence of the $v_{\text {sym }}(\mathrm{N}=\mathrm{N})$ peak at 1525 $\mathrm{cm}^{-1}$, the cis isomer of azobenzene-thiol could be clearly distinguished from the trans isomer. The trans/cis conformational change of ABT molecules after UV light illumination was followed by TER imaging. Furthermore, we discovered that the photoisomerization yield of $A B T$ molecules at $\mathrm{Au}$ grain edges is higher than that on Au terraces, because the cis-to-trans back reaction rate is slower at the grain edges of $\mathrm{Au}$. This is ascribed to the shift of $d$ bands to higher energy and the lower reaction enthalpy of trans-to-cis isomerization at $\mathrm{Au}$ steps, as confirmed by DFT calculation. These results confirm a substrate-mediated charge transfer process for the photoisomerization of $\mathrm{ABT}$ on an Au surface. The ability to spatially distinguish the molecular vibrational features of molecules adsorbed at different surface sites will contribute to a better understanding of the photoisomerization mechanism of photo switches bound to a metal surface.

\section{Acknowledgements}

L.-Q. Z. thanks the Chinese Scholarship Council for a Ph.D. student fellowship. We thank the High Performance Computing Team at ETH Zurich for help with the DFT calculations, Dr. Ewelina Lipiec and Jacek Szczerbinski for helpful discussions, and Dr. Alessandro Lauria for help with recording UV-Vis spectra.

Keywords: photoisomerization • azobenzene thiol $\cdot$ tipenhanced Raman spectroscopy • grain edges • substratemediated charge transfer

[1] H. Qian, S. Pramanik, and I. Aprahamian. J. Am. Chem. Soc., 2017, 139, 9140-9143.

[2] Y. B. Zheng, B. Kiraly, S. Cheunkar. T. J. Huang and P. S. Weiss, Nano Lett., 2011, 11, 2061-2065.

[3] V. Balzani, A. Credi, F. M. Raymo and J. F. Stoddart. Angew. Chem. Int. Ed., 2000, 39, 3349-3391.

[4] J. Robertus, W. R. Browne and B. L. Feringa, Chem. Soc. Rev., 2010, 39, 354-378.

[5] N. Tamai and O.H. Miyasaka, Chem. Rev., 2000, 100, 1875-1890.

[6] H. M. D. Bandara and S. C. Burdette, Chem. Soc. Rev., 2012, 41, 1809-1825.

[7] M. Wolf and P. Tegeder, Surf. Sci., 2009, 603, 1506-1517.

[8] J. Plain, G. P. Wiederrecht, S. K. Gray, P. Royer and R. Bachelot, J. Phys. Chem. Lett., 2013, 4, 2124-2132.

[9] Z. Mahimwalla, K. G. Yager, J. I. Mamiya, A. Shishido, A. Priimagi and C. Barrett, J. Polym. Bull., 2012, 69, 967-1006.

[10] T. J. Kucharski, N. Ferralis, A. M. Kolpak, J. O. Zheng, D. G. Nocera and J. C. Grossman, Nature Chem., 2014, 6, 441-447.

[11] Y. Wang, N. Ma, Z. Wang and X. Zhang, Angew. Chem. Int. Ed., 2007, 46, 2823-2826. 
[12] H. M. Dhammika Bandara and S. C. Burdette, Chem. Soc. Rev., 2012, 41, 1809-1825.

[13] Y. B. Zheng, J. L. Payton and P. S. Weiss et al., Nano Lett., 2011, 11, 3447-3452.

[14] H. Rau and E. Lueddecke, J. Am. Chem. Soc., 1982, 104, 1616-1620.

[15] R. Turanský, M. Konôpka, N. L. Doltsinis, I. Stich and D. Marx, Phys. Chem. Chem. Phys., 2010, 12, 13922-13932.

[16] G. S. Hartley, J. Chem. Soc., 1938, 633-642.

[17] J. Henzl, M. Mehlhorn, H. Gawronski, K. H. Rieder and K. Morgenstern, Angew. Chem., Int. Ed., 2006, 45, 603-606.

[18] R. Schmidt, S. Hagen, D. Brete, R. Carley, C. Gahl, J. Dokc, P. Saalfrank, S. Hecht, P. Tegeder and M. Weinelt. Phys. Chem. Chem. Phys., 2010, 12, 4488-4497.

[19] C. Gahl, D. Brete, F. Leyssner, M. Koch, E. R. McNellis, J. Mielke, R. Carley, L. Grill, K. Reuter, P. Tegeder and M. Weinelt, J. Am. Chem. Soc., 2013, 135, 4273-4281.

[20] N. Tallarida, L. Rios, V. A. Apkarian and J. Lee, Nano Lett., 2015, 15, 6386-6394.

[21] Y. Ootani, K. Satoh, A. Nakayama, T. Noro and T. Taketsugu, J. Chem. Phys., 2009, 131, 194306.

[22] M. Pederzoli, J. Pittner, M. Barbatti and H. Lischka, J. Phys. Chem. A, 2011, 115, 11136-11143.

[23] V. Cantatore, G. Granucci and M. Persico, Comput. Theor. Chem., 2014, 1040-1041, 126-135.

[24] D. P. Hoffman, S. R. Ellis and R. A. Mathies, J. Phys. Chem. A, 2013, 117, 11472-11478.

[25] M. Quick, A. L. Dobryakov, M. Gerecke, C. Richter, F. Berndt, I. N. loffe, A. A. Granovsky, R. Mahrwald, N. P. Ernsting and S. A. Kovalenko, J. Phys. Chem. B, 2014, 118, 8756-8771.

[26] A. L. Dobryakov, M. Quick, I. N. loffe, A. A. Granovsky, N. P. Ernsting and S. A. Kovalenko, J. Chem. Phys., 2014, 140, 184310.

[27] M. Alemani, S. Selvanathan, F. Ample, M. V. Peters, K. H. Rieder, F. Moresco, C. Joachim, S. Hecht and L. Grill, J. Phys. Chem. C, 2008 112, 10509-10514.

[28] X. Wang, S.-C. Huang, T.-X. Huang, H. - S. Su, J.-H. Zhong, Z.-C. Zeng, M.-H. Li and B. Ren, Chem. Soc. Rev., 2017, 2017,46, 40204041.

[29] T. Schmid, L. Opilik, C. Blum and R. Zenobi, Angew. Chem. Int. Ed., 2013, 52, 5940-5954.

[30] G. Ertl, Angew. Chem., Int. Ed., 2013, 52, 52-60

[31] R. M. Stöckle, Y. D. Suh, V. Deckert and R. Zenobi, Chem. Phys. Lett., 2000, 318, 131-136.

[32] M. S. Anderson, Appl. Phys. Lett., 2000, 76, 3130-3132.

[33] N. Hayazawa, Y. Inouye, Z. Sekkat and S. Kawata, Opt. Commun., 2000, 183, 333-336.

[34] B. Pettinger, P. Schambach, C. J. Villagómez and N. Scott, Annu. Rev. Phys. Chem., 2012, 63, 379-399.

[35] H. K. Wickramasinghe, M. Chaigneau, R. Yasukuni, G. Picardi, R. Ossikovski, ACS Nano, 2014, 8, 3421-3426.

[36] T. Schmid, L. Opilik, C. Blum and R. Zenobi, Angew. Chem. Int. Edit., 2013, 52, 5940-5954.

[37] R. Zhang, Y. Zhang, Z. C. Dong, S. Jiang, C. Zhang, L. G. Chen, L. Zhang, Y. Liao, J. Aizpurua, Y. Luo, J. L. Yang and J. G. Hou, Nature, 2013, 498, 82-86.

[38] J.-H. Zhong, X. Jin, L. Meng, X. Wang, H.-S. Su, Z.-L. Yang, C. T. Williams and B. Ren, Nat. Nanotech., 2017, 12, 132-136

[39] G. Pace, V. Ferri, C. Grave, M. Elbing, C.V. Hänisch, M. Zharnikov, M. Mayor, M. A. Rampi and P. Samori, PNAS, 2007, 104, 24, 9937-9942.

[40] M. Hegner, P.Wagner and G. Semenza, Surf. Sci., 1993, 291, 39-46.

[41] J. P. Merrick, D. Moran and L. Radom, J. Phys. Chem. A, 2007, 111, 11683-11700

[42] C. M. Stuart, R. R. Frontiera and R. A. Mathies, J. Phys. Chem. A, 2007 $111,12072-12080$

[43] B. Hammer and J. K. Nørskov, Adv. Catal., 2000, 45, 71-129.

[44] Z.-P. Liu and P. Hu, J. Am. Chem. Soc., 2002, 124, 14770-14779.

[45] J. Stadler, T. Schmid, R. Zenobi, Nano Lett., 2010, 10, 4514-4520. 


\section{Entry for the Table of Contents}

Layout 1:

\section{COMMUNICATION}

The reversible photoisomerization of an azobenzenes-thiol (ABT) self-assembled monolayer was demonstrated by tipenhanced Raman spectroscopy. The cis isomers of $A B T$ were preferentially observed at Au grain edges.

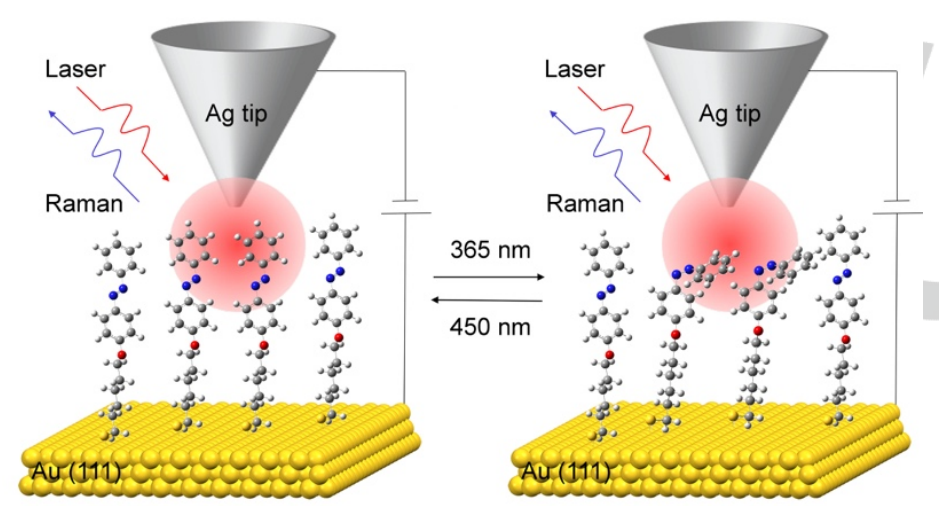

Li-Qing Zheng, Xing Wang, Feng Shao, Martin Hegner, and Renato Zenobi*

Page No. - Page No.

Nanoscale chemical imaging of reversible photoisomerization of an azobenzene-thiol selfassembled monolayer by tip-enhanced Raman spectroscopy 